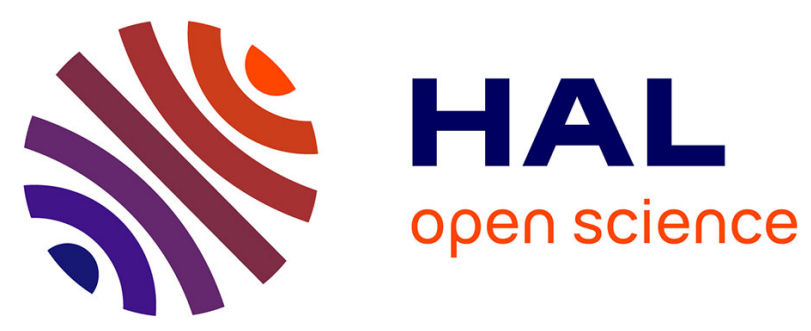

\title{
DONALD MAC FARLANE. - Experiments made to determine surface conductivity for heat in absolute measure (Expériences faites pour déterminer la conductibilité pour la chaleur en mesure absolue); Proceeding's of the Royal Society, t. XX, p. 90
}

\author{
A. Cornu
}

\section{To cite this version:}

A. Cornu. DONALD MAC FARLANE. - Experiments made to determine surface conductivity for heat in absolute measure (Expériences faites pour déterminer la conductibilité pour la chaleur en mesure absolue) ; Proceeding's of the Royal Society, t. XX, p. 90. J. Phys. Theor. Appl., 1873, 2 (1), pp.427-428. 10.1051/jphystap:018730020042700 . jpa-00236907

HAL Id: jpa-00236907 https://hal.science/jpa-00236907

Submitted on 1 Jan 1873

HAL is a multi-disciplinary open access archive for the deposit and dissemination of scientific research documents, whether they are published or not. The documents may come from teaching and research institutions in France or abroad, or from public or private research centers.
L'archive ouverte pluridisciplinaire HAL, est destinée au dépôt et à la diffusion de documents scientifiques de niveau recherche, publiés ou non, émanant des établissements d'enseignement et de recherche français ou étrangers, des laboratoires publics ou privés. 
DONALD MAC FARLANE. - Experiments made to determine surface conductivity for heat in absolute measure (Expériences faites pour déterminer la conductibilité pour la chaleur en mesure absolue); Proceeding's of the Royal Society, t. XX, p. 9o.

L'auteur a déterminé la loi de refroidissement d'une sphère de cuivre dans l'air humide, dans deux cas : la surface étant polie et la surface étant noircic au noir de fumée, le refroidissement s'effectuant dans une enceinte à double paroi remplie d'eau et noircie à l'intérieur.

La température était mesurée par une espèce d'aiguille thermoélectrique dont l'une des soudures était fixée au centre de la sphère ( 2 centimètres de rayon), l'autre en contact avec l'enceinte.

On sait, depuis Dulong et Petit, que la formule deNewton n'est pas applicable aux grandes différences de température, et que, dans la formule différentielle $d \mathrm{Q}=\mathrm{KS} \theta d x=-d \mathrm{C}$, où

$d \mathrm{Q}$ représente la quantité de chaleur émise (calorie);

$\mathrm{K}$ le coefficient de conductibilité extérieure;

$S$ la surface de refroidissement (centimètres carrés)

$\theta$ la différence de température (degrés centigrades);

$x$ le temps (seconde sexagésimale);

C la chaleur spécifique du corps;

la constante $K$ n'est pas invariable et dépend de lavalcur de $\theta$. La réduction des observations de l'auteur porte sur la valeur absolue deK.

Voici le résumé des résultats coordonnés par des formules empiriques :

\begin{tabular}{|c|c|c|c|}
\hline \multirow{2}{*}{$\begin{array}{c}\text { DIFFÉRENCE } \\
\text { de } \\
\text { température. }\end{array}$} & \multicolumn{2}{|c|}{ VALEUR DE $\mathrm{K}$. } & \multirow{2}{*}{$\begin{array}{c}\text { RAPPORT } \\
\text { des pouroirs } \\
\text { émissifs } \\
\text { des deux états } \\
\text { de la } \\
\text { surface. }\end{array}$} \\
\hline & Surface polie. & Surface noircie. & \\
\hline $5^{\circ}$ & 0,000178 & 0,000252 & 0,707 \\
\hline I0 & 0,000186 & 0,000266 & $\circ, 699$ \\
\hline 5 & o, ooor $9^{3}$ & 0,000279 & 0,692 \\
\hline 20 & 0,000201 & 0,000289 & $0,69^{5}$ \\
\hline 25 & 0,000207 & $0,00329^{9}$ & $o, 694$ \\
\hline 30 & 0,000212 & 0,000306 & $0,63^{3}$ \\
\hline 35 & 0,000217 & 0,000313 & $o, 693$ \\
\hline 40 & 0,000220 & 0,0003 I 9 & $o, 693$ \\
\hline 45 & 0,000223 & 0,000323 & 0,690 \\
\hline 50 & 0,000225 & 0,000326 & $\mathbf{o}, 690$ \\
\hline 55 & 0,000226 & 0,000328 & 0,690 \\
\hline $6 o$ & 0,000226 & 0,000328 & $\mathbf{o}, 690$ \\
\hline
\end{tabular}

3 r. 
Ces nombres vérificnt la conclusion de Dulong et Petit, à savoir que les vitesses de refroidissement ne dépendent de l'état des surfaces que par une constante de proportionnalité.

L'accélération négative du rapport des pouvoirs émissifs n'infirme pas sensiblement cette conclusion; clle est si faible qu'elle peut être attribuée à une petite erreur régulière dans l'évaluation des différences de température : en effet, l'auteur ne parait tenir aucun compte d'une cause délicate d'erreur qui avait préoccupé Dulong et Petit, à savoir la résistance inégale à la transmission de la chalcur dans les deux cas. Il est évident que, dans le refroidissement le plus rapide, la température est distribuée moins uniformément que dans le cas d'un refroidissement lent; l'aiguille thermoélectrique indique donc moins bien la température moyenne de la masse que les boules de mercure des physiciens français.

Sauf ces réserves, les recherches de l'auteur sont d'un bon exemple, non-seulement pour établir la concordance des méthodes, mais surtout pour engager les physiciens à se préoccuper, plus qu'on ne le fait d'ordinaire, des déterminations en valeur absolue, si favorables aux études ultérieures.

A. Cornu. 